01.5

\title{
Метод определения характеристик перемежающейся обобщенной синхронизации на основе расчета локальных показателей Ляпунова
}

\author{
(c) О.И. Москаленко ${ }^{1,2}$, Е.В. Евстифееев ${ }^{1,2}$, А.А. Короновский ${ }^{1,2}$ \\ ${ }^{1}$ Саратовский национальный исследовательский государственный университет им. Н.Г. Чернышевского, Саратов, Россия \\ ${ }^{2}$ Региональный научно-образовательный математический центр „Математика технологий будущего“, Саратов, Россия \\ E-mail: o.i.moskalenko@gmail.com
}

Поступило в Редакцию 27 апреля 2020г.

В окончательной редакции 27 апреля 2020г.

Принято к публикации 12 мая 2020г.

\begin{abstract}
Предложен метод выделения ламинарных и турбулентных фаз в связанных системах, находящихся вблизи границы обобщенной хаотической синхронизации, основанный на расчете локальных показателей Ляпунова. Работоспособность метода проверена на системах с однонаправленной связью, допускающих анализ перемежаемости при помощи метода вспомогательной системы. Результаты указанных методов сопоставлены друг с другом, получено хорошее согласие между ними.
\end{abstract}

Ключевые слова: обобщенная синхронизация, локальные показатели Ляпунова, метод вспомогательной системы, перемежаемость.

DOI: 10.21883/PJTF.2020.16.49846.18359

Обобщенная синхронизация является одним из наиболее интересных типов хаотической синхронизации, привлекающих пристальное внимание исследователей, работающих в различных областях науки и техники $[1,2]$. Интерес к этому явлению помимо фундаментальных аспектов обусловлен прежде всего его большой практической значимостью. В частности, известно, что именно режим обобщенной синхронизации может найти применение в информационно-телекоммуникационных системах, в частности, при разработке способов скрытой передачи информации, обладающих высокой устойчивостью по отношению к шумам и помехам в канале связи [3,4].

Под обобщенной хаотической синхронизацией $[1,5]$ в контексте потоковых динамических систем понимают существование функциональной связи (функционала) между состояниями этих систем. Выделяют однонаправленную и взаимную связь между системами $[5,6]$.

В случае однонаправленной связи между взаимодействующими осцилляторами на границе обобщенной синхронизации наблюдается перемежающееся поведение - режим перемежающейся обобщенной синхронизации $[7,8]$. В данном случае во временно́й динамике систем синхронная динамика (ламинарные фазы) прерывается асинхронными всплесками (турбулентными фазами), в то время как в режиме обобщенной синхронизации турбулентные всплески отсутствуют.

Для анализа характеристик перемежающейся обобщенной синхронизации используется, как правило, метод вспомогательной системы [9,10]. Согласно этому методу, необходимо ввести в рассмотрение вспомогательную систему, идентичную одной из взаимодействующих систем (ведомой системе) по управляющим параметрам, и проанализировать разность между состояниями этих систем в разные моменты времени. В режиме перемежаемости, как отмечалось выше, эта разность представляет собой чередование ламинарных и турбулентных фаз, проанализировав статистику которых, можно определить тип перемежаемости, реализуемый в исследуемой системе. Известно, что на границе обобщенной синхронизации в однонаправленно связанных системах с достаточно простой топологией аттрактора наблюдается перемежаемость типа „оn-off“ [7], характеризующаяся степенными законами для распределений длительностей ламинарных фаз

$$
N(\tau) \sim \tau^{-3 / 2}
$$

и зависимостью средней длительности ламинарных фаз от параметра надкритичности

$$
\langle\tau\rangle \sim\left(\varepsilon_{c}-\varepsilon\right)^{-1},
$$

где $\varepsilon_{c}-$ граница обобщенной синхронизации. Для систем с взаимным типом связи определить тип перемежаемости не представляется возможным в связи с неработоспособностью метода вспомогательной системы в данном случае [11], что указывает на необходимость разработки универсальных методов выделения характерных фаз поведения систем, находящихся вблизи границы обобщенной синхронизации, справедливых как в случае однонаправленной, так и взаимной связи.

В настоящей работе предложен метод выделения ламинарных и турбулентных фаз, основанный на расчете локальных показателей Ляпунова [12], и проведена его апробация на однонаправленно связанных трехмерных динамических системах. Согласно данному подходу, необходимо провести расчет показателей Ляпунова [13] в ограниченном временно́м диапазоне и исследовать временну́ю динамику второго (в случае однонаправленной связи - старшего условного) показателя Ляпунова. 


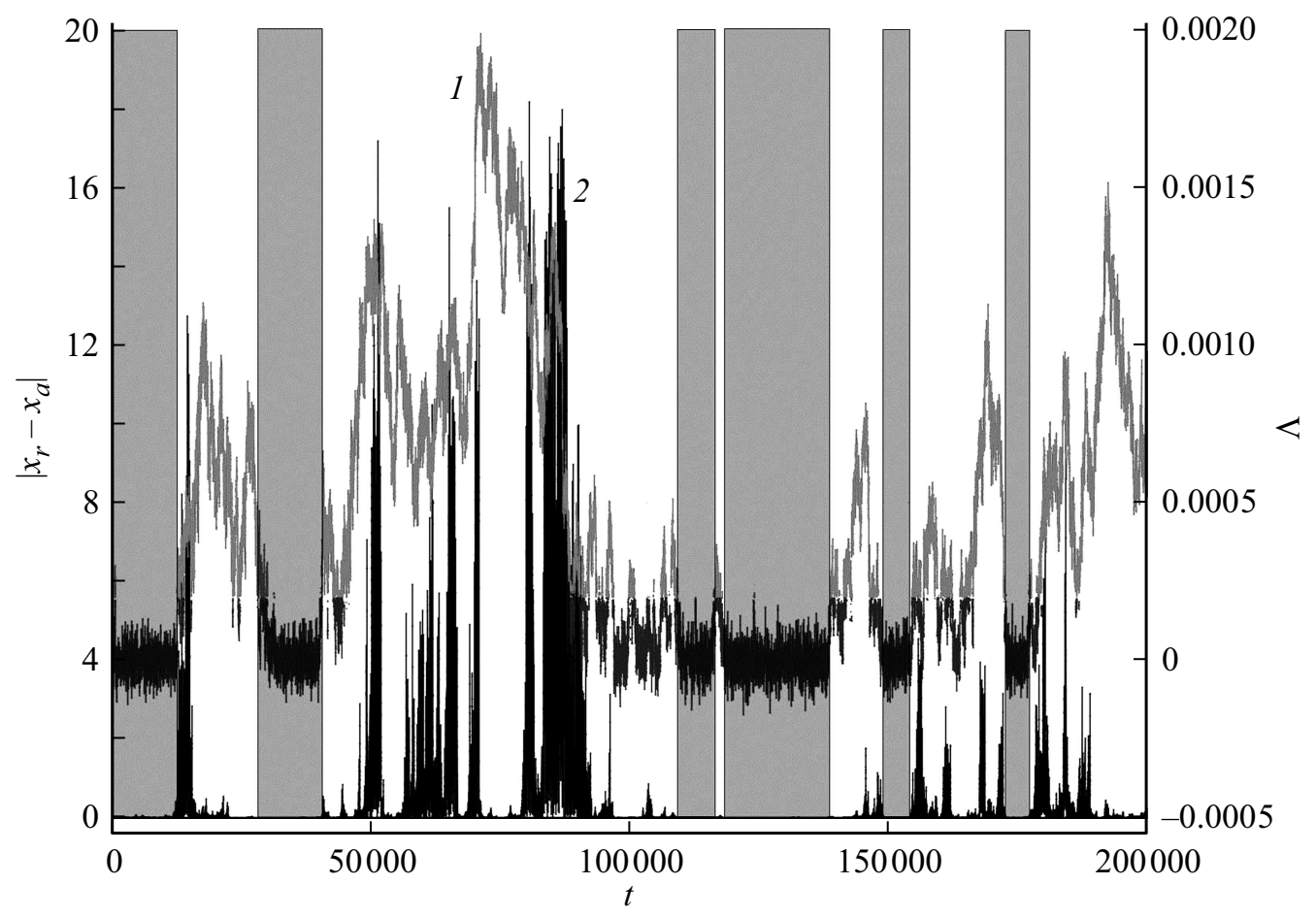

Рис. 1. Временны́е зависимости старшего условного локального показателя Ляпунова, рассчитанного для системы двух однонаправленно связанных осцилляторов Ресслера (3) (правая вертикальная ось, линия 1), и временна́я динамика абсолютной величины разности между $x$-координатами ведомой и вспомогательной систем (левая вертикальная ось, линия 2). Значение параметра связи $\varepsilon=0.109$. Старший условный локальный показатель Ляпунова рассчитывался на временно́м интервале $T=3500$. Области, соответствующие ламинарным фазам поведения, отмечены серыми прямоугольниками.

По характеру данной зависимости можно однозначно идентифицировать наличие ламинарных и турбулентных фаз.

В качестве объекта исследования выбраны однонаправленно связанные системы Ресслера [7], описываемые следующей системой уравнений:

$$
\begin{gathered}
\dot{x}_{d}=-\omega_{d} y_{d}-z_{d}, \\
\dot{y}_{d}=\omega_{d} x_{d}+a y_{d}, \\
\dot{z}_{d}=b+z_{d}\left(x_{d}-c\right), \\
\dot{x}_{r}=-\omega_{r} y_{r}-z_{r}+\varepsilon\left(x_{d}-x_{r}\right), \\
\dot{y}_{r}=\omega_{r} x_{r}+a y_{r}, \\
\dot{z}_{r}=b+z_{r}\left(x_{r}-c\right),
\end{gathered}
$$

где индексы $d$ и $r$ относятся к ведущей и ведомой системам соответственно, $a=0.15, b=0.2, c=10$, $\omega_{d}=0.99, \omega_{r}=0.95-$ управляющие параметры, $\varepsilon-$ параметр связи. При выбранных значениях управляющих параметров режим обобщенной синхронизации в системе (3) наступает при критическом значении $\varepsilon_{c}=0.11$.

Ниже границы обобщенной синхронизации, как уже отмечалось, наблюдается перемежающееся поведение. Для определения статистических характеристик перемежаемости были использованы как метод вспомогательной системы, так и подход, основанный на вычислении локальных показателей Ляпунова. На рис. 1 представлены фрагменты временны́х зависимостей старшего условного локального показателя Ляпунова $\Lambda$, рассчитанного для системы (3) при $\varepsilon=0.109$, и модуля разности состояний ведомой и вспомогательной систем Ресслера $\left|x_{r}-x_{a}\right|$ при том же значении параметра связи. Видно, что в моменты времени, соответствующие турбулентным фазам поведения, и разность состояний ведомой и вспомогательной систем, и величина локального показателя Ляпунова оказываются положительными, тогда как во время ламинарных фаз разность состояний ведомой и вспомогательной систем оказывается близкой к нулю, а локальный показатель Ляпунова - близким к нулю либо отрицательным. Такое соответствие между поведением ведомой и вспомогательной систем и локальными показателями Ляпунова свидетельствует о возможности применения обоих методов для определения статистических характеристик длительностей ламинарных фаз в режиме перемежаемости, имеющей место вблизи границы обобщенной синхронизации.

На рис. 2, 3 представлены рассчитанные обоими способами статистические характеристики длительностей ламинарных фаз: распределения длительностей ламинарных фаз при нескольких значениях параметра связи (рис. 2) и зависимости средних длительностей ламинарных фаз от параметра надкритичности $\left(\varepsilon-\varepsilon_{c}\right)$ (рис. 3). Данные численного моделирования показаны 


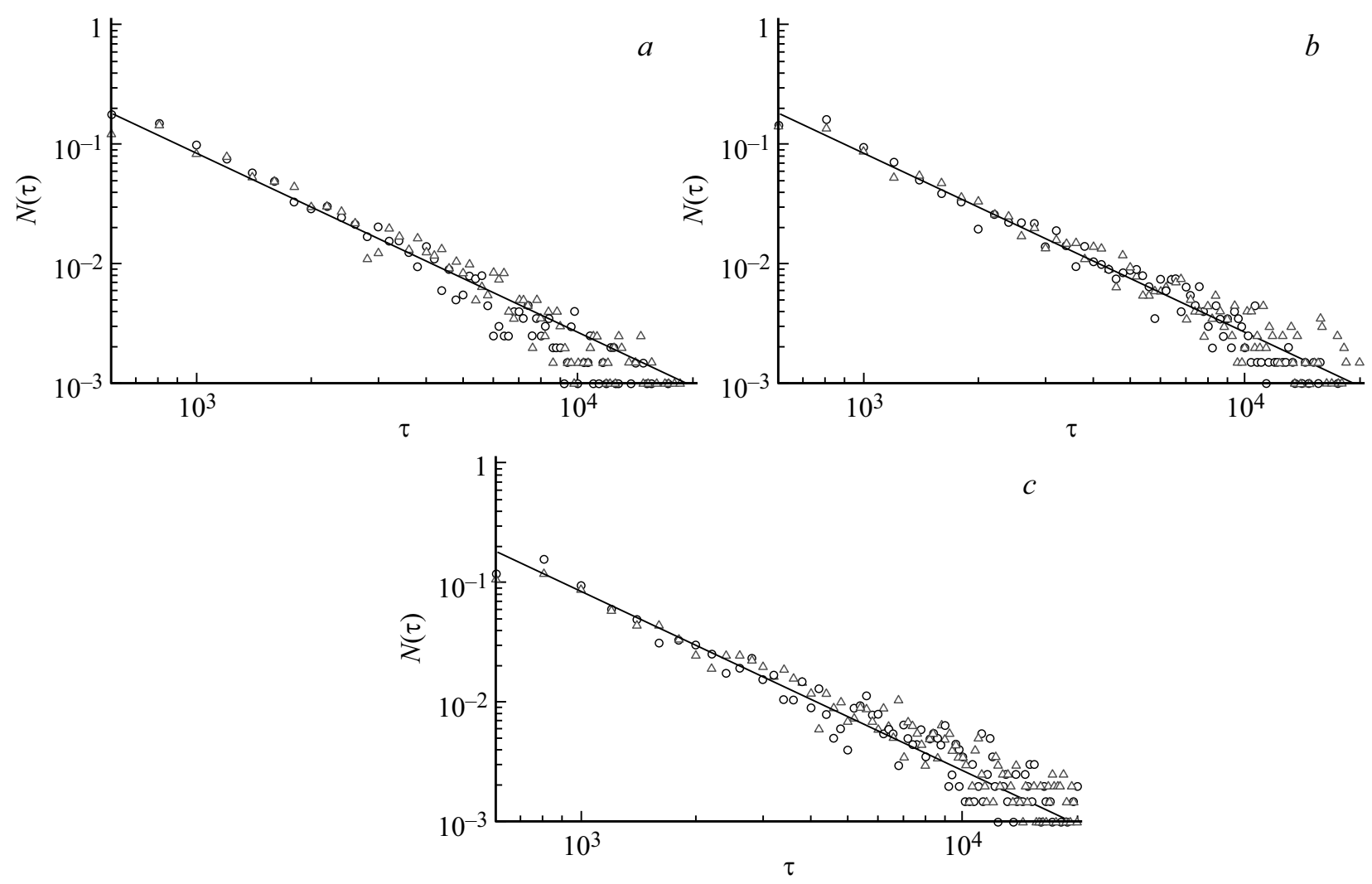

Рис. 2. Нормированные распределения длительностей ламинарных фаз двух однонаправленно связанных систем Ресслера (3), рассчитанные при помощи метода вспомогательной системы (кружки) и использовании локальных показателей Ляпунова (треугольники), и их аппроксимации степенным законом (1), полученные при фиксированных значениях параметра связи $\varepsilon=0.107(a), 0.108(b)$ и $0.109(c)$.

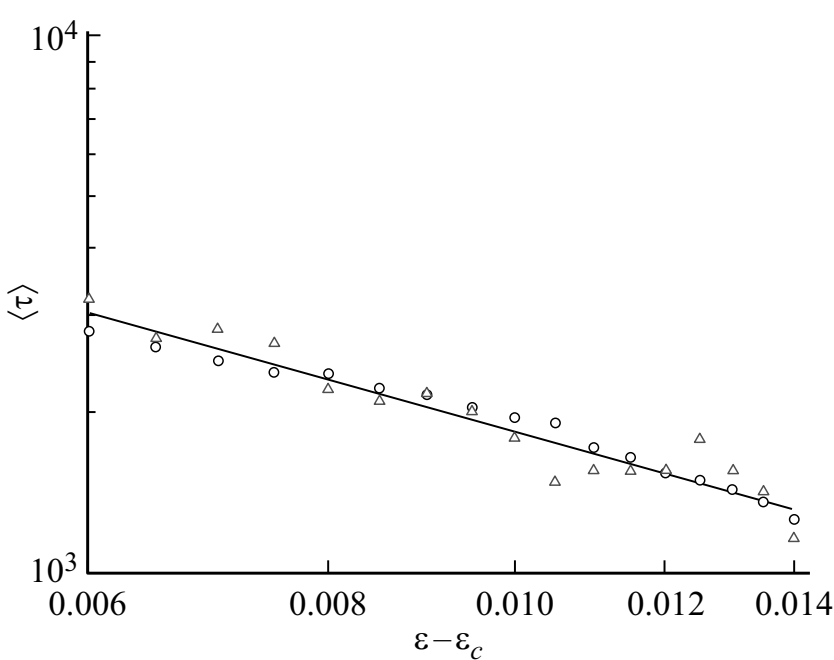

Рис. 3. Зависимости средних длительностей ламинарных фаз от параметра надкритичности $\left(\varepsilon-\varepsilon_{c}\right)$, полученные при помощи метода вспомогательной системы (кружки) и использовании локальных показателей Ляпунова (треугольники), и их аппроксимации степенным законом (2).

символами, теоретические аппроксимации - сплошными линиями. Из рисунков видно, что во всех рассмот- ренных случаях статистические характеристики, полученные двумя различными способами, практически в точности совпадают друг с другом, что указывает на возможность использования локальных показателей Ляпунова для определения статистических характеристик перемежаемости. Можно ожидать, что предложенный подход окажется применимым и в том случае, когда применение метода вспомогательной системы не представляется возможным, например в случае взаимной связи между системами [6,14]. Кроме того, нетрудно заметить, что полученные статистические характеристики длительностей ламинарных фаз подчиняются степенным законам (1) и (2) в четком соответствии с теорией перемежаемости типа „on-off“.

Таким образом, в работе предложен метод выделения ламинарных и турбулентных фаз, основанный на вычислении локальных показателей Ляпунова, и проведено его сопоставление с методом вспомогательной системы. Показано, что оба метода позволяют однозначно определить статистические характеристики перемежаемости в случае однонаправленной связи между системами, что свидетельствует о возможности применения предложенного подхода даже в том случае, когда традиционные методы и подходы оказываются неработоспособными. 


\section{Финансирование работы}

Работа выполнена при финансовой поддержке Российского научного фонда (проект № 19-12-00037).

\section{Конфликт интересов}

Авторы заявляют, что у них нет конфликта интересов.

\section{Список литературы}

[1] Rulkov N.F., Sushchik M.M., Tsimring L.S., Abarbanel H.D.I. // Phys. Rev. E. 1995. V. 51. N 2. P. 980-994. DOI: 10.1103/PhysRevE.51.980

[2] Стародубов А.В., Короновский А.А., Храмов А.Е., Жарков Ю.Д., Дмитриев Б.С. // Письма в ЖТФ. 2007. Т. 33. B. 14. C. 58-65.

[3] Terry J.R., Van Wiggeren G.D. // Chaos Solitons Fractals. 2001. V. 12. P. $145-152$.

DOI: 10.1016/S0960-0779(00)00038-2

[4] Короновский А.А., Москаленко О.И., Храмов А.Е. // ЖТФ. 2010. T. 80. B. 4. C. $1-8$.

[5] Koronovskii A.A., Moskalenko O.I., Hramov A.E. // Phys. Rev. E. 2011. V. 84. N 3. P. 037201.

DOI: 10.1103/PhysRevE.84.037201

[6] Moskalenko O.I., Koronovskii A.A., Hramov A.E., Boccaletti S. // Phys. Rev. E. 2012. V. 86. N 3. P. 036216. DOI: 10.1103/PhysRevE.86.036216

[7] Hramov A.E., Koronovskii A.A. // Europhys. Lett. 2005. V. 70. N 2. P. $169-175$. DOI: $10.1209 / \mathrm{epl} / \mathrm{i} 2004-10488-6$

[8] Попов П.В. // Письма в ЖТФ. 2007. Т. 33. В. 18. С. 61-69.

[9] Abarbanel H.D.I., Rulkov N.F., Sushchik M. // Phys. Rev. E. 1996. V. 53. N 5. P. 4528-4535.

DOI: 10.1103/PhysRevE.53.4528

[10] Короновский А.А., Москаленко О.И., Сельский А.О. // Письма в ЖТФ. 2020. Т. 46. В. 7. С. 48-51. DOI: 10.21883/PJTF.2020.07.49221.18126

[11] Moskalenko O.I., Koronovskii A.A., Hramov A.E. // Phys. Rev. E. 2013. V. 87. N 6. P. 064901. DOI: 10.1103/PhysRevE.87.064901

[12] Короновский А.А., Куровскал М.К., Храмов А.Е., Шурыгина C.A. // ЖТФ. 2009. Т. 79. В. 10. С. 1-9. DOI: $10.1134 / \mathrm{S} 1063784209100016$

[13] Pyragas K. // Phys. Rev. E. 1997. V. 56. N 5. P. 5183-5188. DOI: 10.1103/PhysRevE.56.5183

[14] Москаленко О.И., Ханадеев В.А., Короновский А.А. // Письма в ЖТФ. 2018. Т. 44. В. 19. С. 87-95. DOI: $10.21883 /$ PJTF.2020.16.49846.18359 